\title{
Platelet Depletion/Transfusion as a Lethal Factor in a Colitis-associated Cancer Mouse Model
}

\author{
ANASTASIOS ANGELOU $^{1}$, EFSTATHIOS ANTONIOU ${ }^{1}$, ANASTASIA PIKOULI ${ }^{2}$, \\ JANE WANG $^{3}$, MARCO VENTIN ${ }^{4}$, STEFAN BUETTNER ${ }^{3}$, MUHAMMAD FAATEH ${ }^{3}$, \\ GEORGE THEODOROPOULOS ${ }^{5}$, GEORGIOS C. ZOGRAFOS $^{5}$, STAMATIOS THEOCHARIS $^{6}$, \\ APOSTOLOS E. PAPALOIS ${ }^{7,8}$, EMMANOUIL PIKOULIS ${ }^{2}$ and GEORGIOS ANTONIOS MARGONIS ${ }^{3}$ \\ ${ }^{1}$ Second Department of Propedeutic Surgery, Laiko General Hospital, Medical School, \\ National and Kapodistrian University of Athens, Athens, Greece; \\ ${ }^{2} 3 r d$ Department of Surgery, School of Medicine, National and Kapodistrian University of Athens, \\ "Attikon" University General Hospital, Athens, Greece; \\ ${ }^{3}$ Department of Surgery, Johns Hopkins University School of Medicine, Baltimore, MD, U.S.A.; \\ ${ }^{4}$ Department of Surgery, University of Udine, Udine, Italy; \\ ${ }^{5}$ First Department of Propaedeutic Surgery, Hippokratio General Hospital of Athens, \\ Medical School, National and Kapodistrian University of Athens, Athens, Greece; \\ ${ }^{6}$ First Department of Pathology, National and Kapodistrian University of Athens, Athens, Greece; \\ ${ }^{7}$ Experimental, Educational and Research Centre ELPEN, Athens, Greece; \\ ${ }^{8}$ Medical School, European University Cyprus, Nicosia, Cyprus
}

\begin{abstract}
Background/Aim: TLR-4 Knock-out (KO) mice are protected from colitis-associated cancer in the established AOM/DSS mouse model. The aim of this study was to assess whether the TLR4 KO mice would still be protected from carcinogenesis after platelet depletion and transfusion with TLR4 wild-type platelets. Materials and Methods: Thirty-two female C57BL6 mice were divided into 6 groups. Among the three groups that received Azoxymethane/Dextran Sulfate Sodium (AOM/DSS), one group included TLR4KO mice, which were depleted of their platelets and were then transfused with platelets from TLR4 wild-type mice. The other two groups included wild-type and TLR-4KO mice that only received AOM/DSS. Results: All 6 animals in the $K O$ group that underwent platelet depletion/transfusion succumbed. Three of them died before the administration of DSS and three in the week following DSS administration. In contrast, mice in the other two groups experienced less weight loss and only 1 mouse died in each of them. Conclusion: Platelet depletion/transfusion
\end{abstract}

Correspondence to: Georgios Antonios Margonis, Division of Surgical Oncology, Department of Surgery, Johns Hopkins University, Medical School, Pre-Clinical Teaching Building (PCTB), 725 N Wolfe St (East Monument Street), Baltimore, MD 21205, U.S.A. Tel: +1 4434401103, e-mail: antonis.margonis@gmail.com

Key Words: Platelet depletion, colitis, mice. was detrimental in TLR-4 transgenic mice that received AOM/DSS.

Patients with inflammatory bowel disease (IBD) are at risk of developing colitis-associated colorectal cancer (CAC) (1), which differs from sporadic colorectal cancer. CAC development is a multi-step process, from the initial inflammation to overt malignancy (2). One of the experimental models that mimics the development of CAC uses a combination of azoxymethane (AOM) and Dextran sulfate sodium (DSS) (3). Specifically, the mutagen AOM is administered intraperitoneally as a single dose and DSS is administered to mice via drinking water and induces a chronic inflammatory state. AOM induces mutations and DSS accelerates the process of tumor formation from several months to only a few weeks $(3,4)$. It has been postulated that increased levels of sCD40L play an important role in the pathogenesis of CAC (5). It is also known that TLR4 signaling is crucial for colon carcinogenesis, and that its inhibition may be useful in the prevention of colitisassociated cancer (6). A possible link between sCD40L levels and TLR-4 is platelets, as platelets express TLR-4 and secrete $\mathrm{sCD} 40 \mathrm{~L}$ when activated.

Based on these findings, we aimed to deplete TLR4 knock-out (KO) mice, which received AOM/DSS, of their platelets (PLT) and then transfuse them with platelets from TLR4 wild-type mice. The aim of the study was to assess whether the TLR4 KO mice would still be protected from 
carcinogenesis after platelet depletion and transfusion with TLR4 wild-type platelets.

\section{Materials and Methods}

Experimental conditions, animals, and employed chemicals. TLR4-/mice ( 8 weeks old) were purchased from Oriental Bio Service, Inc. (Kyoto, Japan). All knockout mice were backcrossed to C57BL/6 mice for at least 8 generations. C57BL/6 wild-type mice were purchased from the Hellenic Pasteur Institute. All animals were maintained at the ELPEN Animal Facility, in compliance with Institutional Animal Care Guidelines and EU regulations (directive 63/2010). All animals were housed in plastic cages (6 mice/cage) with free access to drinking water and a pelleted basal diet, under controlled conditions of humidity $(50 \pm 10 \%)$, light $(12 / 12$ hour light/dark cycle) and temperature $\left(23 \pm 2^{\circ} \mathrm{C}\right)$. The experimental animals were quarantined for the first 7 days, and were then randomly placed into experimental and control groups. The colonic carcinogen AOM was purchased from Sigma Chemical Co. (St. Louis, MO, USA), while DSS was purchased from ICN Biochemicals, Inc (Aurora, OH, USA).

Experimental protocol. A total of 32 mice were divided into 5 groups (3 experimental groups and 2 control groups) of 6 in each group and a group of 2 mice. Specifically, groups $1(n=6)$ and $2(n=6)$ were the control groups (wild type C57BL6 and TLR4 Knock Out, respectively). Group 3 included 2 wild-type mice that underwent only platelet depletion/transfusion without following the AOM/DSS protocol.

Groups $4(n=6)$ and $5(n=6)$ were treated with a single intraperitoneal injection of AOM $(10 \mathrm{mg} / \mathrm{kg}$ of body weight). In Group 4, all mice were wild type C57BL6 and in Group 5, all mice were genetically modified TLR4 Knock Out mice. According to the protocol, one week following AOM administration, the animals received $2.5 \%$ DSS in their drinking water for 7 days, followed by a 2-week rest period without DSS, then another 7-day cycle of $2.5 \%$ DSS followed by a second 2 -week rest period, that ended with a final 7-day cycle of $2.5 \%$ DSS.

Group $6(n=6)$ included TLR4 KO mice that on day 1 were treated with a single intraperitoneal injection of a commercially available rat antimouse GPIb $\alpha$ monoclonal antibody ( $4 \mu \mathrm{g} / \mathrm{g}$ of body weight, Emfret Analytics, Eibelstadt, Germany) for platelet depletion, and then received intraperitoneal $\mathrm{AOM}(10 \mathrm{mg} / \mathrm{kg}$ of body weight $)$ three days later. One week following AOM administration, the animals received $2.5 \%$ DSS in their drinking water for 7 days. According to the protocol, every 7 days all mice were platelet depleted, and were then transfused with platelets 3 days later.

For platelet counts, mice were bled from the retro-orbital plexus under isoflurane anaesthesia (IsoFlo, Abbott Laboratories, Abbott Park, IL). Blood was collected into a heparin-containing tube and mixed with $10 \mathrm{mM}$ (final concentration) EDTA as an anticoagulant. Platelet counts in whole blood were analyzed using a HEMAVET HV950FS multispecies haematology analyser (The Americas Drew Scientific Inc, Oxford, CT). For platelet transfusion, $1 \times 109$ washed platelets resuspended in $0.2 \mathrm{ml}$ saline were injected into the lateral tail vein for each recipient mouse.

\section{Results}

Although the original endpoint of the study concerned the long-term effects (i.e. protection of carcinogenesis) of platelet depletion and subsequent transfusion (with TLR4 wild-type platelets) among TLR4 KO mice, herein we report the short-term outcomes of the experiment. Specifically, we report the results of the experiment until the 18th day, counting from the day that TLR-4 KO mice were platelet depleted for the first time. This time point was selected because at this time none of the platelet depleted TLR-4 KO mice (Group 6) were alive.

In Group 6, all mice developed loose stools (with gross evidence of blood) during DSS administration and experienced extensive weight loss. None of the mice recovered during the water-only rest period, and as a result, all mice died due to rectal bleeding and extensive weight loss. During the final histopathologic examination, all mice had developed severe colitis and massive rectal bleeding. Specifically, platelet depletion started 3 days before AOM administration, and at the time of AOM injection, all mice in this group were in good health. Three days following AOM injection, the first mouse succumbed due to profuse rectal bleeding and a body weight reduction from $21.79 \mathrm{gr}$ to $19.70 \mathrm{~g}$. One week later, when DSS was to be added to the drinking water, we noticed two additional losses due to rectal bleeding. At that time, the first mouse had a body weight reduction of $0.4 \mathrm{~g}$ (from 19.83 to $19.52 \mathrm{~g}$ ), and the second mouse had a body weight reduction of $2 \mathrm{~g}$ (from 21.79 to $19.70 \mathrm{~g}$.). These two mice had received the second dose of platelet depletion three days before their death. On day 8 , we continued with the administration of DSS in the drinking water. One week later, the remaining three mice died due to massive rectal hemorrhage and substantial weight loss. We noticed that mouse EII weighted $17.68 \mathrm{~g}$ on day 8 of the experiment, and one week later weighted $13.50 \mathrm{~g}$. The weights of the other two mice are referred to in Table I.

It is worth noting that the mice of Group 1 and Group 2 were in good health at the second week of the experiment and continued to do well until the end of the procedures on day 18 . Of note, the 2 mice in group 3 were in good health until the end of the procedures on day 18 .

In Group 4, which had the wild type mice that received AOM/DSS, there was one death during the first week of the experiment. Specifically, the mouse succumbed 3 days following the intraperitoneal administration of AOM due to peritonitis. In addition, this mouse experienced significant weight loss, from 19.31 to $18.01 \mathrm{~g}$. During the second week of the experiment and following the administration of DSS in the drinking water, the remaining mice had a mean weight reduction of $10 \%$ of their body weight (Table II).

During the first week of the procedures and five days following the administration of AOM, another mouse died in group 5. Specifically, this mouse died on the 5th day, and signs of peritonitis were found on autopsy. 
Table I. Procedures and body weights (grams) in Group 5.

\begin{tabular}{|c|c|c|c|c|c|c|c|}
\hline TLR4KO AOM/DSS PLT & E0 & EI & EII & EIII & EIIII & E5 & \\
\hline -3 Day & 20.65 & 19.83 & 19.42 & 19.05 & 20.30 & 21.79 & PLT Depl. \\
\hline 1st Day & 20.81 & 20.86 & 20.39 & 19.58 & 20.48 & 21.51 & AOM+PLTs \\
\hline 3rd Day & 20.45 & 20.42 & 19.00 & 18.35 & 18.04 & 19.70 & \\
\hline 5th Day & 19.09 & 19.52 & 17.20 & 17.30 & 18.18 & & PLT Depl. \\
\hline 8th Day & 20.63 & & 17.68 & 17.06 & & & DSS+PLTs \\
\hline 10th Day & 20.70 & & 18.40 & 17.72 & & & DSS \\
\hline 12th Day & 18.47 & & 15.23 & 17.16 & & & DSS+PLT Depl. \\
\hline 15th Day & & & 13.50 & & & & PLTs \\
\hline
\end{tabular}

PLT Depl.: Platelets depleted; AOM: azoxymethane; DSS: dextran sulfate sodium.

Table II. Procedures and body weights (grams) in Group 3.

\begin{tabular}{|c|c|c|c|c|c|c|c|}
\hline WT AOM/DSS & Г० & $\Gamma \mathrm{I}$ & $\Gamma \mathrm{II}$ & ГIII & ГIIII & $\Gamma 5$ & \\
\hline -3 Day & 21.82 & 20.61 & 21.14 & 20.31 & 20.32 & 19.31 & \\
\hline 1st Day & 21.71 & 20.50 & 18.56 & 20.66 & 20.50 & 20.12 & $\mathrm{AOM}$ \\
\hline 3rd Day & $21 / 71$ & 19.87 & 19.80 & 17.00 & 19.32 & 18.01 & \\
\hline 5th Day & 21.40 & 16.53 & 20.40 & 15.25 & 19.32 & & \\
\hline 8th Day & 21.96 & 22.95 & 21.87 & 20.67 & 21.24 & & DSS \\
\hline 10th Day & 21.35 & 21.17 & 20.88 & 20.68 & 20.96 & & DSS \\
\hline 12th Day & 21.20 & 20.44 & 20.99 & 19.00 & 21.18 & & DSS \\
\hline
\end{tabular}

AOM: Azoxymethane; DSS: dextran sulfate sodium.

\section{Discussion}

This is a short communication of a study, which used the AOM/DSS model of colitis-associated cancer in TLR-4 KO mice transfused with wild-type PLT following PLT depletion for the first time. Unfortunately, all 6 mice succumbed to overwhelming intestinal inflammation. We report this finding for two reasons. Firstly, researchers in the field of intestinal carcinogenesis should be aware of the high risk of death when applying this model. Secondly, there are two interesting plausible explanations. First, it is possible that the depletion/transfusion procedure itself is responsible for the worse outcomes due to procedure-related stress. Second, the unique genetic background of the transfused animals (silenced for TLR-4 cell types with the exception of the wild-type PLT) may be responsible for the worse outcomes in this group of mice.

Regarding the former model, the two wild-type mice of group 3 that underwent the platelet depletion/transfusion procedure were in good health until the end of the procedures on day 18. As such, it appears that the depletion/transfusion procedure per se may not be responsible for the worse outcomes, despite the procedure-related stress.

Regarding the latter, although AOM negatively impacts the wellbeing of wild-type mice, it mostly exerts a long-term effect on tumorigenesis $(3,7)$. Indeed, in the wild-type mice that received AOM, we only noticed a modest impairment of their wellbeing compared to the negative controls, and only one animal died. In contrast, $\mathrm{KO}$ mice that were transfused with wild-type PLT experienced much worse weight loss, and three of the six mice died. As such, the deterioration in the clinical condition of the KO mice cannot be attributed to the wild-type PLT, as this was common in the two groups. Instead, it may be attributed to the interplay of AOM with their mixed backgrounds: TLR-4 KO cells with only PLT being wild-type.

The next question is whether this interplay and the poor outcomes in the transfused animals are specific to AOM. In the next stage of the experiment, all groups of mice received DSS. Again, the KO mice that were transfused with wildtype platelets had much worse clinical outcomes. They suffered greater weight loss, and in fact, all three succumbed after the first cycle of DSS. In contrast, the wild-type mice suffered modest weight loss and only one of the six died. Given that only DSS was administered repeatedly (AOM was only administered once at the beginning of the experiment), it appears that the worse outcomes in the $\mathrm{KO}$ mice that were transfused with wild-type PLT were not AOM specific, but rather reflective of the unique background of wild-type PLT among TLR-4 KO cells. 
The fact that DSS worsened the outcomes of both wildtype and KO mice, albeit to a different extent, is consistent with previous studies. For example, Yu et al. (8) have reported that wild-type platelet depletion decreases tissue damage and clinical disease activity index in DSS-treated animals. Specifically, colitis was induced by oral administration of 5\% DSS for 5 days in female Balb/c mice. In the histopathologic specimen, it was found that the morphological features were improved in colitis mice depleted of wild-type platelets, and more specifically, mucosal ulcerations were absent, crypt architecture was restored, and the only finding was the presence of inflammatory cells. In accordance with these results, Sato et al. (9) have induced colitis with 3\% DSS for 6 days in C57BL/6J mice. The depletion of their wild-type PLT ameliorated murine colitis.

This study has some limitations. First, the number of the mice in each group was relatively small. However, the clinical deterioration and lethality in the transfused animals was universal. Furthermore, the cost of purchasing TLR4KO mice is high, and most previous studies that used them also have relatively small groups (6). Second, we did not test whether wild-type mice would have a worse outcome after being transfused with PLT from TLR-4 KO mice, which would have been an interesting parallel to the fact that we transfused KO mice with wild-type PLT.

This study is important practically because it is the first to demonstrate the detrimental effects of wild-type platelet transfusion in TLR-4 KO mice. The latter model is widely used in research and thus our results can be used in future studies. Future studies should explore the hypothesis that the observed detrimental effects might be attributed to the presence of wild-type platelets in the context of all other cell types being TLR-4 KO.

\section{Conflicts of Interest}

None.

\section{Authors' Contributions}

AA, EA and GAM were responsible for the conception and design, analysis and interpretation for the data, drafting and critically revising the article, and of the final approval of the version to be published. AP, JW, MV, SB and MF were responsible for the analysis and interpretation of data, critically revising the article and for the final approval of the version to be published. GT, GCZ, ST, AEP and EP were responsible for the conception and design, analysis and interpretation of data, critically revising the article, final approval of the version to be published.

\section{Acknowledgments}

This study was funded by the Experimental, Educational and Research Center ELPEN as Research Scholarship. The authors would like to thank A. Zacharioudaki, M.A. Tsoutsou, K. Tsarea, E. Karampela, M. Karamperi, N. Psychalakis, A. Karaiskos, S. Gerakis, and E. Gerakis, staff members of the Experimental, Educational and Research Center ELPEN, for their invaluable assistance during the experiments.

\section{References}

1 Grivennikov SI: Inflammation and colorectal cancer: colitisassociated neoplasia. Semin Immunopathol 35: 229-244, 2013. PMID: 3568220. DOI: 10.1007/s00281-012-0352-6

2 Shanahan F: Review article: colitis-associated cancer -- time for new strategies. Aliment Pharmacol Ther 18(Suppl 2): 6-9, 2003. PMID: 12950414.

3 Neufert C, Becker C and Neurath MF: An inducible mouse model of colon carcinogenesis for the analysis of sporadic and inflammation-driven tumor progression. Nat Protoc 2: 19982004, 2007. PMID: 17703211. DOI: 10.1038/nprot.2007.279

4 Tanaka T: Preclinical cancer chemoprevention studies using animal model of inflammation-associated colorectal carcinogenesis. Cancers (Basel) 4: 673-700, 2012. PMID: 3712717. DOI: $10.3390 /$ cancers 4030673

5 Cognasse F, Hamzeh-Cognasse H, Lafarge S, Delezay O, Pozzetto B, McNicol A and Garraud O: Toll-like receptor 4 ligand can differentially modulate the release of cytokines by human platelets. Br J Haematol 141: 84-91, 2008. PMID: 18279456. DOI: 10.1111/j.1365-2141.2008.06999.x

6 Fukata M, Chen A, Vamadevan AS, Cohen J, Breglio K, Krishnareddy S, Hsu D, Xu R, Harpaz N, Dannenberg AJ, Subbaramaiah K, Cooper HS, Itzkowitz SH and Abreu MT: Tolllike receptor-4 promotes the development of colitis-associated colorectal tumors. Gastroenterology 133: 1869-81, 2007. PMID: 2180834. DOI: $10.1053 /$ j.gastro.2007.09.008

7 Parang B, Barrett CW and Williams CS: AOM/DSS model of colitis-associated cancer. Methods Mol Biol 1422: 297-307, 2016. PMID: 5035391. DOI: 10.1007/978-1-4939-3603-8_26

8 Yu C, Zhang S, Wang Y, Zhang S, Luo L and Thorlacius H: Platelet-derived CCL5 regulates CXC chemokine formation and neutrophil recruitment in acute experimental colitis. J Cell Physiol 231: 370-376, 2016. PMID: 26089223. DOI: 10.1002/ jcp.25081

9 Sato H, Higashiyama M, Hozumi H, Sato S, Furuhashi H, Takajo T, Maruta K, Yasutake Y, Narimatsu K, Yoshikawa K, Kurihara C, Okada Y, Watanabe C, Komoto S, Tomita K, Nagao S, Miura $\mathrm{S}$ and Hokari R: Platelet interaction with lymphatics aggravates intestinal inflammation by suppressing lymphangiogenesis. Am J Physiol Gastrointest Liver Physiol 311: G276-85, 2016. PMID: 27313177. DOI: 10.1152/ajpgi.00455.2015

Received March 7, 2019

Revised March 30, 2019

Accepted April 3, 2019 